\title{
Dénomination de groupes sociaux : approche sémantique et discursive d'une catégorie de noms propres
}

\author{
Lecolle, Michelle \\ Université de Lorraine, CREM-Praxitexte \\ Michelle.lecolle@univ-lorraine.fr
}

\begin{abstract}
Cet article se centre sur les dénominations de groupes sociaux constitués (partis politiques, syndicats, associations, groupes culturels - Parti Radical, Confédération paysanne, Génération précaire, Orchestre de Radio France), en les décrivant dans leur singularité, d'un point de vue sémantique et formel et du point de vue de leur emploi en discours. Ce faisant, et par là même, c'est aussi la question de leur statut théorique qui est abordée. En effet, si ces dénominations sont généralement considérées comme des noms propres (Npr désormais), ce qui est aussi notre option, elles n'en partagent pas, du moins à première vue, toutes les propriétés. D'abord pour ce qui concerne leur référent, qui est ici un collectif humain, un groupe constitué ; ensuite du point de vue de leur mode de construction : ces noms sont basés sur du matériau lexical, et ils sont souvent polylexicaux, ce qui implique qu'ils ont une syntaxe interne et qu'ils peuvent être descriptifs. Ces deux aspects s'articulent à un troisième, qui tient à la spécificité du référent : contrairement aux anthroponymes et aux toponymes, ces noms sont forgés pour dénommer le groupe dont ils accompagnent la création - même il s'agit d'une simplification, nous partons de ce postulat. Se pose dès lors la question du sens de ces noms ou, du moins, de leur transparence descriptive vis-à-vis de leur référent, par exemple en tant que groupe (Parti, Union, Fédération, Syndicat, Association, Cercle, Ligue), en tant que groupe de telle catégorie ou ayant telle caractéristique (socialiste, de gauche, de la magistrature, ouvrier - Lutte Ouvrière, Force Ouvrière), ou encore de tel domaine, et rassemblé selon tel objectif (politique, social, culturel - Parti vs Orchestre vs Syndicat, etc.)... si transparence il y a toutefois. En effet, tous les Npr de groupes sociaux ne sont pas descriptifs, ou ne le sont pas de la même manière : le nom du groupe musical Fauve, par exemple, formé sur un signe de la langue française (substantif ou adjectif), ne dit rien de son référent, du point de vue du collectif, de l'humain ni, apparemment du moins, du point de vue de la musique. S'il y a une descriptivité, celle-ci n'est pas d'un accès immédiat, du moins pour les non-initiés - ce qui n'empêche pas qu'il y ait motivation, mais sur d'autres bases.
\end{abstract}

Enfin, comme pour les Npr en général, la forme de ces noms a une incidence sur leur emploi en discours : d'un point de vue morphosyntaxique, on entrevoit cette incidence si l'on compare des Npr de groupes avec ou sans article (Les Économistes atterrés vs Médecins du Monde), commençant ou non par un nom (Secours Populaire vs Sauvons les Riches), selon le type de nom (Droit (au logement), Secours (Populaire) vs Ligue/Parti/Cercle de [...]); ou encore si l'on compare, en termes d'accord, un Npr de groupe pluriel (les Verts, par exemple) ou singulier ((le) Parti Socialiste). La comparaison de ces deux noms (syntagme explicitement pluriel vs syntagme avec nom collectif) laisse aussi envisager des conséquences en termes d'appréhension du groupe (comme un ensemble d'individus, ou comme un tout global). Sur le plan discursif, encore, la forme de certains de ces Npr rend nécessaire si ce n'est indispensable la présence d'un support syntactico-sémantique nominal (un nom collectif - Ncoll désormais) plus ou moins spécifique permettant de les introduire, ou simplement de les insérer en discours : (le) collectif Jeudi Noir/Fauve, (l')association Médecins du Monde, (le) mouvement les/des Indignés, (le) groupe Indochine/Fauve, (le) syndicat Solidaires/Autonomes. Mais ceci non pas indépendamment de leur notoriété, puisque le Npr de groupe connu peut se passer d'introducteur.

Nous rassemblons les dénominations des groupes sociaux sous le terme de «Npr collectifs » (Npr-coll), en nous centrant sur les propriétés qui rapprochent ces objets linguistiques : référent collectif et humain, rassemblement délibéré des membres du groupe (qu'il soit contemporain ou hérité de l'histoire), caractère 
proprial de la dénomination, et ceci en dépit de leurs différences - de forme (monolexicale vs polylexicale ; descriptive $v s$ non, ou moins, descriptive), de domaine (politique, culturel, social etc.) et de notoriété.

Concernant l'ensemble des Npr-coll, les phénomènes présentés nous paraissent interdépendants, et ce sont ces fils enchevêtrés que nous nous proposons de démêler ici. Notre première partie délimite la catégorie des Npr-coll et en propose un inventaire en plusieurs types. Dans une deuxième partie, nous discutons le statut proprial des Npr-coll, et nous nous attardons, à propos de certaines dénominations (les Indignés), sur le rapport entre stabilisation du référent en tant que groupe et stabilisation en $\mathrm{Npr}$ de l'expression qui sert à le dénommer. À cette fin, nous reprenons de Bosredon (1997), et (notamment) de Veniard (2009) et Calabrese (2010) la distinction entre «nom propre» et «dénomination propre ». Pour notre problématique, l'emploi de l'expression «dénomination propre» nous permettra de signaler les étapes de ce qui apparait de fait comme un processus, entre désignation libre émergente et cristallisation en Npr-coll. Une troisième partie se centre sur les enjeux discursifs liés aux Npr-coll : choix et utilisation du nom, dans différents emplois. Dans cette partie, nous examinerons le rapport de motivation formeréférent à travers plusieurs exemples de Npr-coll. Cette question, potentiellement infinie, traverse en réalité l'ensemble de l'article, puisqu'elle est engagée par l'objet linguistique étudié lui-même, et par le point de vue sémantique et discursif adopté. Nous entendrons par «motivation», du moins dans un premier temps, le fait très général de l'existence d'un lien non arbitraire entre forme et «contenu » des Npr étudiés. Cette notion peut ensuite se préciser en termes de caractère compositionnel et/ou descriptif du matériau lexical qui fournit les Npr-coll. Mais la question se pose aussi d'une autre manière : des Nprcoll comme Jeudi Noir, les Têtes Raides, Indochine, ou La Bourgeoise sont interprétables de par leur matériau lexical, mais peut-on les dire motivés en tant que Npr, et par rapport à quoi ?

Comme pour les Npr en général, avec ceux-ci peut-être plus encore, la description des Npr-coll engage et mêle inextricablement des questions de langue, de discours, de monde et d'encyclopédie. Nous en prenons acte, sans (trop) nous aventurer en dehors de notre terrain linguistique, mais en cherchant à repérer ce qui, de cet « extra-linguistique », vient interférer avec le linguistique, et comment il influe.

\section{Groupes et noms de groupes}

Afin de cerner les enjeux de la description des noms de groupes constitués, nous rappelons tout d'abord quelques éléments concernant les Ncoll en général et les Ncoll de groupes sociaux constitués plus particulièrement. Une deuxième section propose un inventaire de Npr-coll. Dans cette partie, nous «faisons comme si » la caractérisation de ces dénominations en termes de nom propre était acquise, ce qui sera discuté plus loin $(\S 2)$.

\subsection{Des noms collectifs humains aux noms propres institutionnels}

Les particularités des Ncoll ont été décrites à propos des noms communs (Nc désormais). En voici une définition :

Un Ncoll est un nom au singulier dénotant une entité composée d'un regroupement d'éléments de même catégorie ${ }^{1}$

Outre les traits définitoires que sont le singulier morphologique et la pluralité sémantique interne, la définition souligne la similitude des membres du groupe (" éléments de même catégorie ») et l'existence d'un niveau supérieur, celui du groupe lui-même (« entité »). Les groupes dénotés par les Ncoll peuvent être rassemblés selon diverses modalités - similitude ou origine (bouquet, domesticité, fratrie), proximité spatiale (foule, chambrée, forêt), point de vue valorisant ou dévalorisant (élite, racaille), but commun (chœur, orchestre, association, comité, parti) etc. Les Ncoll qui nous intéressent ici relèvent de cette dernière catégorie, qui ne concerne que les groupements humains : ce regroupement est fondé sur un lien social et une finalité commune, un «faire ensemble », plus ou moins délimité - les types de groupes (partis, syndicats etc.) présentés en $\S 1.2$ en fournissent des exemples. 
La sémantique des Ncoll, et plus encore celle de ces noms de groupes sociaux, est marquée par l'existence du double niveau mentionné. Une des conséquences en est que les éléments et le groupe sont, tout à la fois, reliés et distincts. En effet, le groupe peut être créé en tant que tel à partir d'éléments existants mais épars - comme en (1) où est présentée l'amorce de la constitution d'un groupe (cf. le Ncoll mouvement), groupe qu'il est également nécessaire de nommer, fût-ce provisoirement (cf. port. precarios inflexiveis) :

(1) Voilà trois mois que les precarios inflexiveis, "précaires inflexibles », ont posé leurs valises au [+ adresse], sur les hauteurs de Lisbonne [...]. Mieux qu'un siège, ils ont ouvert un bar associatif [...] «Sans lieu, sans existence physique, notre mouvement ne tiendrait pas longtemps. Notre café lui permet d'avoir un visage ». (Télérama, à propos du Portugal, 12/2012)

Le groupe peut aussi changer, dans une certaine mesure, sans que les individus ne changent; et inversement les individus peuvent changer sans que l'entité-groupe ne change, comme dans l'exemple qu'utilise Thomas Hobbes ${ }^{2}$, parlant de : «la même cité, dont les actes procèdent continuellement de la même institution, que les habitants soient les mêmes ou non ». Ce jeu de permanence/changement et de différences entre groupe constitué et ensemble d'individus se vérifie sur le plan discursif si l'on observe qu'on peut sans contradiction prédiquer, à propos des référents des Ncoll en tant que groupes, des propriétés qui ne sont pas vraies des individus les composant ${ }^{3}$. On peut ainsi affirmer, par exemple, que Solidaires, le Front de Gauche et Droit au Logement étaient présents à telle table ronde alors même que ces groupes n'y étaient que représentés ; ou encore, malgré l'absurdité apparente, énoncer (2) :

(2) Les Verts ne sont pas morts, ils se transforment. (Radio, 11/2010, à propos de la fusion avec Europe Écologie)

Créés et perpétués selon une finalité donnée, nos groupes sociaux doivent se donner un nom, afin d'acquérir une existence, juridique et publique (selon leur finalité, et dans un cercle plus ou moins étroit). Bacot (2010) remarque, à propos des Npr de partis, que «les baptêmes en politique sont le plus souvent datables et localisables » : de fait, la création du Npr accompagne généralement la création officielle du groupe lui-même - et la re-création du Npr accompagne le changement, voire la transformation du groupe (voir les exemples de LCR/NPA, CNPF/MEDEF, Médecins sans frontières/Médecins du Monde ${ }^{4}$ ). Ainsi, si le groupe dont il est question en (1) connaît une certaine longévité, on peut également supposer que la dénomination precarios inflexiveis (ou une autre) en viendra à se figer, et à se fixer à ce référent collectif.

Enfin, dernière caractéristique des noms de groupes sociaux, à mettre en lien avec la nomination du groupe : si, à l'instar de Nc ordinaires, les Ncoll association, syndicat, parti, orchestre, chœur désignent une classe (celle des associations, des syndicats, des partis etc.) et sont, à ce titre, pluralisables, les noms de groupes constitués et officialisés désignent, eux, des entités uniques (l'Association des Sciences du Langage, le Parti de Gauche, le Syndicat de la magistrature, la Fondation Concorde). Par ailleurs, comme signalé ci-dessus, leur référent collectif possède un statut « institutionnel » : il est connu et reconnu dans une société donnée (ce qui se traduit, de manière plus ou moins centrale ou anecdotique, par différents éléments de représentation et d'affichage : logo, porte-parole, site internet), où il joue un rôle (plus ou moins central selon la catégorie de référent : parti vs groupe musical), à l'instar, cette fois, d'entités institutionnelles comme la police, la justice, la magistrature ou encore le parlement, le Sénat etc. C'est pour rendre compte de ces différentes propriétés que nous caractérisons les Npr-coll en termes de « Ncoll institutionnels » (voir Lecolle à paraitre a).

En résumé, ce que nous appelons «Npr-coll» possède les propriétés sémantiques et référentielles suivantes : ce sont des dénominations collectives ; leur référent est composé de personnes (des membres, des adhérents) regroupées selon un but commun; ce groupe humain a un statut d'institution (au sens large). Quant à la forme des Npr-coll, nous y revenons ci-dessous. 


\subsection{Inventaire de noms propres collectifs}

Notre collecte de Npr-coll comporte essentiellement des Npr «français » (utilisés en France et dont le matériau lexical appartient au français), et quelques traductions en français de $\mathrm{Npr}$ (groupes sociaux ou politiques étrangers) lorsqu'elles sont en usage. En dehors des Npr de partis, que nous avons relevés systématiquement, l'inventaire n'est pas exhaustif - nous avons simplement collecté plusieurs exemples de chacune des catégories, en privilégiant les Npr contemporains et utilisés sur la scène publique ${ }^{5}$. Du point de vue linguistique, nous avons cherché à faire figurer, une certaine diversité de formes de Npr mais aussi, le cas échéant, les proximités de forme (Croix-Rouge/Croissant-Rouge; Secours populaire/catholique; SNES/SNESUp ; Médecins sans Frontières/Médecins du Monde). Pas d'exhaustivité donc, mais parler de «représentativité » ne serait pas non plus exact, en particulier pour les noms d'associations, ou de groupes culturels, trop nombreux. Comme signalé en introduction, nous opérons un rassemblement des Npr-coll sur leurs propriétés communes - ce qui entraine que nous ne nous attardons pas sur certaines de leurs différences.

Notre présentation se base sur une catégorisation d'ordre extra-linguistique : les types de groupes (parti politique, syndicat, association etc.) sont distingués selon le mode de rassemblement des membres et l'objectif de constitution du groupe, telle qu'il est affiché publiquement ${ }^{6}$. Cette répartition en types de groupes, faite par commodité de présentation, ne laisse pas préjuger de sa pertinence sur le plan linguistique.

Nous excluons de notre objet les Npr d'entreprises (privées ou publiques) même s'ils sont proches des Npr-coll à de nombreux égards (leurs référents sont des entités institutionnelles). Cependant, il ne s'agit pas réellement selon nous de Ncoll humains, là aussi pour des raisons extra-linguistiques : les employés et salariés ne sont pas à proprement parler des membres des entreprises, mais dépendants de celles-ci, ce qui a des incidences sur l'emploi des noms en discours ${ }^{7}$. Nous excluons également les noms de fédérations et d'organismes institutionnels (Organisation des Nations Unies, Organisation Mondiale du Commerce, Ligue Arabe, etc.), dans la mesure où leurs référents ne sont pas directement constitués d'humains, et sont plutôt des « groupes de groupes » (groupements de pays par exemple). Bien que la ressemblance avec les Npr-coll étudiés soit grande (ce sont aussi des Ncoll et des Npr), nous préférons en rester à la spécificité de «l'humain », avec les conséquences qui en découlent : création du groupe, choix du nom de ce groupe, utilisation du nom pour référer à des humains et non à la seule institution. Enfin, une autre catégorie de Npr partage des caractéristiques sémantico-référentielles avec les Npr-coll présentés ici : il s'agit des Npr de lieux habités (villes, pays), qui prennent usuellement un sens collectif en contexte, comme nous l'avons montré ailleurs (Cislaru et Lecolle 2010, Lecolle à paraitre a, à paraitre b). Ces noms, dans leur acception collective, sont bien marqués par «l'humain» (citoyenneté et habitat commun), mais ils n'ont pas toutes les caractéristiques qui nous occupent ici : ce sont d'abord des Npr de lieu, donc leur sens n'est pas fondé directement sur la mise en œuvre d'un but commun, et le Npr est « hérité » et non pas créé par les membres du groupe ${ }^{8}$.

Quelques remarques sur la collecte: nous présentons des noms d'organismes existants, ou encore présents dans l'histoire récente (Ligue Communiste Révolutionnaire, par exemple). La remarque s'applique aux Npr de partis, mais aussi aux groupes musicaux et plus généralement culturels, et peut s'appliquer aux associations. Par ailleurs, bien que les Npr-coll, en particulier les $\mathrm{Npr}$ de partis et de syndicats, soient plutôt connus siglés $(C G T)$, nous les présentons et les décrivons sous leur forme « déployée » (Confédération Générale du Travail), puisque c'est précisément cette forme qui nous occupe. D'ailleurs c'est bien cette forme qui est interrogée dans les cas où un sigle n'est pas connu comme, par exemple, dans le discours de presse lorsqu'intervient un nom d'organisation nouveau ou supposé peu connu : s'il est siglé (3) ou en langue étrangère (4) et (1), le texte présente sa «traduction». Cette pratique, outre qu'elle fait partie des règles d'écriture du discours journalistique, a aussi la fonction de (re-)donner à la dénomination son/un sens descriptif, ce qui permet de catégoriser ou de qualifier le référent, ou de le rapporter à une éventuelle motivation.

(3) S. M., du Gisti (Groupe d'information et de soutien des immigrés), espère que la nouvelle procédure pourra aboutir et déterminera des responsabilités. (Libération, 18/06/2013) 
(4) Qui sont ces miliciens d'Al-Shabbaab qui ont organisé l'assaut meurtrier du centre commercial Westgate Mall de Nairobi ? [...] Pour comprendre qui sont ces combattants et d'où ils viennent, il faut remonter à 2006 [...] C'est à ce moment que l'Union des tribunaux islamiques se scinde, donnant naissance à Al-Shabbaab ( $\underline{\text { la }}$ jeunesse »). (Politis, 26/09/2013, après l'attaque d'un centre commercial de Nairobi)

Par ailleurs, et bien que ce ne soit pas le propos central, on ne peut pas ignorer que certains phénomènes se jouent parfois dans le sigle ou dans le rapport entre sigle et nom : sous le sigle $U M P$, qui est toujours la forme employée, se sont glissés successivement, en quelques années, Union pour la majorité présidentielle et Union pour un mouvement Populaire; d'un tout autre point de vue : un acronyme tel que CAP21 semble, avec le Nc cap, porter son propre message, différent de celui de Citoyenneté Action Participation pour le $21^{\text {ème }}$ siècle; il en est de même pour Solidaires, Unitaires, Démocratiques / Sud.

1. Noms de partis 9

Centre national des Indépendants et Paysans (CNI(P)); Chasse, Pêche, Nature et tradition (CPNT) ; Citoyenneté Action Participation pour le 21ème siècle (CAP21); Europe Écologie les Verts (EELV); Front National (FN) ; Jeunesses nationalistes révolutionnaires (JNR); Mouvement Jeunes Communistes de France (MJCF); Les Verts; Ligue Communiste Révolutionnaire (LCR); Lutte Ouvrière (LO) ; Mouvement Démocratique (Modem) ; Mouvement pour la France (MPF) ; Mouvement Républicain et Citoyen (MRC) ; Le Nouveau Centre; Nouveau Parti anticapitaliste (NPA) ; Parti Communiste (PC) ; Parti de Gauche (PG); Parti des Travailleurs (à l'étranger: Brésil, Algérie, Tunisie) ; Parti Ouvrier Indépendant (POI) ; Parti radical (PR) ; Parti Radical de Gauche (PRG) ; Parti Socialiste (PS) ; Union des Démocrates et Indépendants (UDI) ; Union pour un mouvement Populaire (UMP).

2. Noms de syndicats

Nous rassemblons quelques noms de syndicats interprofessionnels (Force Ouvrière, UNSA, CGT), et d'autres liés à des corporations. Parmi ceux-ci, certains affichent cette appartenance (Syndicat de la magistrature, Union des Étudiants de France, Mouvement des Entreprises de France ${ }^{10}$ etc.), et d'autres non (Alliance).

Alliance (syndicat de police) ; Autonomes ; Confédération française démocratique du travail (CFDT) ; Confédération Générale du Travail (CGT) ; Confédération paysanne ; Force Ouvrière ; Mouvement des entreprises de France (MEDEF); Solidaires, Unitaires, Démocratiques (Sud); (Union Syndicale) Solidaires; Syndicat national des enseignements du second degré (SNES); Syndicat National de l'Enseignement Supérieur (SNESup) ; Syndicat de la Magistrature ; Synergie Officiers (police).

3. Noms d'associations, d'ONG, d'organisations

Il s'agit ici de noms de groupes, officiels ou non, intervenant sur la scène publique sur le plan social ou politique de manière plus ou moins affichée, parfois de manière contestataire - ce qu'on appelle parfois « la société civile ». Cette catégorie est quelque peu disparate d'un point de vue référentiel. Nous tentons un regroupement en deux sous-ensembles (regroupement «politique», au sens large vs social ou caritatif), en gardant à l'esprit le fait que la frontière entre eux est poreuse, voire artificielle (où caser, par exemple, Amnesty International ?) et qu'elle n'a sans doute pas réellement de pertinence sur le plan linguistique. Ce qui rapproche les référents de ces noms, c'est le fait que le regroupement de leurs membres est ou a été opéré selon un but explicite qui n'est ni professionnel ni politicien (par opposition aux noms de partis et de syndicats).

Nous joignons quelques noms de groupes, qui ne sont connus (ou n'existent ?) qu'en anglais, et qui ont ou ont acquis une notoriété et une portée internationale. Du point de vue linguistique, les questions de lisibilité compositionnelle nous paraissent similaires en anglais et en français.

(les) Anonymous ; Association pour la taxation des transactions financières et pour l'action citoyenne (ATTAC) ; Cercle des Économistes ; Droit au Logement (DAL) ; Fondation Copernic ; Fondation Terra Nova; Génération précaire; Institut Montaigne; Jeudi Noir; (Les) Économistes Atterrés; Les Indignés ; Ligue des droits de l’homme ; Occupy Wall Street; Que choisir ? Réseau Éducation Sans 
Frontière (RESF); Robin des Toits (Association Nationale pour la sécurité Sanitaire dans les technologies sans fil) ; Sauvons les Riches ; Sauvons l'Université.

Action contre la faim; Amnesty International ; Association des Sciences du Langage (ASL) ; CroixRouge; Croissant-Rouge; Emmaüs; (Les) Restos du cour; Médecins du Monde; Médecins sans Frontières; Secours catholique; Secours populaire; Société française d'ethnomusicologie; Union nationale des associations de parents, de personnes handicapées mentales et de leurs amis (Unapei).

Certains Npr-coll ont une forme très productive, dont nous n'avons fait figurer que quelques exemples : [association/société/union/ligue (+ localisation) + pour + objectif / de + appartenance].

4. Noms de groupes culturels

À la différence des autres groupes, les membres des groupes culturels ne sont pas des adhérents, mais des « participants ». En dépit de cette différence, nous considérons les noms de groupes culturels comme des Npr-coll : ils en ont les autres caractéristiques (cf. § 1.1). Au relevé de noms de groupes contemporains, nous ajoutons pour mémoire des noms d'ensembles et d'orchestres classiques, et de compagnies de danse. Les noms de groupes culturels se caractérisent par une certaine liberté formelle. On trouve cependant certaines régularités (des SN définis pluriel : Les Innocents, The Beatles).

Chanson plus bifluorée ; Fauve; Indochine ; Intermède (chorale) ; Les Innocents ; (Les) Petits chanteurs à la croix de bois ; Têtes raides (originellement Red Ted) ; The Beatles ; The Doors ; Tryo ; Zebda.

Les dénominations de formations plus classiques sont, au contraire, normées : les syntagmes

[Jazz Band/Orchestre (philarmonique/symphonique) + nom de lieu]

ou [Ensemble/Trio/Chœur + expansion (Npr ou Nc épithète/complément de nom)]

sont des formes régulières et reproductibles.

Chœurs de l'Armée Rouge ; Ensemble Variances ; Orchestre de radio France; Orchestre philarmonique de Berlin ; Caroline/Louisiana Jazz Band ${ }^{11}$; Trio Wanderer ; Chronos quartet.

Enfin, les quelques dénominations de troupes de danse relevées sont construites autour d'un Npr de personne : Béjart Ballet Lausanne ; Compagnie Pietragalla.

Cet inventaire, si succinct soit-il, nous donne quelques bases concrètes pour aborder, du point de vue de la motivation, la forme des Npr-coll (voir $\S 3$ ). Nous emploierons à ce propos le terme de « signalétique », proposé par Bosredon (1997 : 231-266) d'abord à propos des titres de tableaux. Ce terme, qui renvoie à un «ensemble de marques linguistiques" s'appliquant à "un domaine spécifique de référents » (Bosredon 2011 : 169), présente l'intérêt d'engager «l'imbrication entre langue, discursivité et pratiques sociales » (Veniard 2009), qui est fondamentale aussi pour notre objet. Il serait pertinent, peut-être, d'envisager une description différenciée de la signalétique de chacune de nos catégories, mais nous n'entreprenons pas ici ce (gros) travail, nous contentant de faire référence, par la suite, à l'une ou l'autre des catégories.

\section{Dénomination de groupes sociaux et nom propre}

Les études sur le Npr abordent peu, du moins spécifiquement, la question de la dénomination d'entités collectives, bien que leur statut de Npr soit généralement plus ou moins implicitement admis ${ }^{12}$. Pourtant, l'intégration dans les Npr de ces dénominations collectives ne va pas de soi, et ceci pour deux raisons :

(i) à la différence des Npr généralement invoqués et considérés comme prototypiques (toponymes et anthroponymes), ils dénotent un groupe, donc une pluralité d'éléments $(\S 2.1)$;

(ii) leur forme est (souvent) compositionnelle et, par voie de conséquence, (souvent) descriptive. Du coup, on ne peut pas vraiment les dire «vides de sens », ce qui pose des problèmes au regard de certaines théories du $\mathrm{Npr}(\S 2.2)$. 
Nous explorons ces points dans ce qui suit. Nous nous basons sur la définition du Npr proposée par Jonasson $(2004: 21)$ : « toute expression associée dans la mémoire à long terme à un particulier en vertu d'un lien dénominatif conventionnel stable ». En le formulant autrement, on peut aussi parler de «contrat de dénomination » : le Npr est fixé par un «acte de baptême » (Kripke [1972] 1982), et, à partir de là, contractuellement transmis et reconnu. Nous proposons en 2.3 une conclusion quant aux questions posées, et envisageons le cas intermédiaire des dénominations de groupes non stabilisés.

\subsection{Dénomination d'entités collectives}

Proposer une définition qui parle de lien dénominatif à un particulier peut paraître contradictoire pour les Npr-coll. Mais cette contradiction n'est qu'apparente, si l'on se réfère à la description du Ncoll proposée en $\S 1.1$, qui mentionne deux niveaux : dans ce cadre, le Npr est le nom du groupe social lui-même.

Pas de contradiction, donc, mais des spécificités : on ne peut en effet laisser de côté le fait que ce particulier est constitué d'une pluralité interne. Ce double statut a des incidences : en discours, le Npr-coll peut référer à l'un ou l'autre des niveaux différemment, ou être ambigu. Il faudrait approfondir et illustrer cet aspect des choses ${ }^{13}$, ou encore la question (signalée brièvement en 1.1) de la représentation du groupe par un élément saillant, qui se pose spécifiquement à propos des dénominations de groupes institutionnels, mais nous laissons de côté ces points pour nous attarder sur un autre aspect, celui de ce que nous nommerons le «pouvoir rassembleur» de la dénomination. Si, en effet, toute dénomination possède 1'«indéniable pouvoir démiurgique [de faire exister une chose pour nous] 》(Frath 2010), ce pouvoir s'exerce doublement pour les Npr-coll : non seulement la dénomination s'applique à un groupe et participe à son institutionnalisation, mais encore, plus fondamentalement, elle consacre la transformation d'un ensemble d'individus en groupe social. Autrement dit, dès lors qu'un groupe, fût-il informel, reçoit une dénomination, il en reçoit ipso facto une forme de reconnaissance en tant que groupe : les precarios de (1), les Indignés, ou encore, avec une autre forme, « les » Occupy (Wall Street), comme il est parfois dit, sont de bons exemples de tels processus où des individus se constituent ainsi en groupe sous nos yeux - par les médias. Passer de cette étape de dénomination plus ou moins stable à celle où on peut réellement parler de Npr-coll revient à parler de la stabilisation du groupe, du point de vue de sa constitution même, et du point de vue de sa reconnaissance par son entour. Pour d'autres groupes sociaux plus organisés (partis politiques mais aussi, par exemple, groupes culturels), la création et l'affichage explicites du Npr sont la version officielle de ce même processus.

\subsection{Forme des dénominations de groupes sociaux et « sens »}

Le fait que les noms soient créés pour dénommer une entité elle-même créée (un artefact) distingue les Npr-coll des Npr prototypiques, mais les rapproche des noms d'œuvres (littéraires, cinématographiques, picturales). D'un autre point de vue, les Npr-coll étant généralement descriptifs, ils se rapprochent d'autres Npr descriptifs, ceux du type Jardin des Plantes, rue de Rivoli, qualifiés de Npr «mixtes » par Jonasson (op. cit.). Cette dimension descriptive des Npr-coll entraine des conséquences du point de vue de leur statut, discutable, de Npr.

Sans reprendre ici le débat sur le Npr vide ou plein de sens qui occupe de nombreux écrits ${ }^{14}$, précisons dans quels termes se pose la question pour notre objet : il ne s'agit pas ici d'un sens correspondant à une ou des «descriptions définies » substituables ou attachées au nom (Frege et Russell, cités par Kleiber 1981), ni d'un sens qui serait la description du référent sous la forme d'un faisceau de propriétés (Searle 1972). Dans ces approches d'ordre logique ou sémantico-pragmatique, le terme de description renvoie au rapport Npr-référent (référent-Npr, plutôt) et non à la forme, simple ou composée, du Npr. Inversement, ce qui nous intéresse ici est le caractère descriptif ou, plus généralement, motivé du Npr et donc le sens éventuellement attaché au signe lui-même, dans son rapport au référent - ce qui distingue, dans les termes de Gardiner ([1953] 2005) puis de Jonasson (op. cit.), les Npr «purs » (totalement arbitraires) vs «non purs » (Jardin des Plantes, Mont Blanc). Ce rapport de motivation a, lui aussi, été discuté par certains auteurs, pour être récusé : à partir de l'exemple du Mont Blanc chez Mill ([1843] 2005), de la ville de Dartmouth chez Mill, Gardiner et Kripke, du New College à Oxford et de Peak's Tunnel (Coates 2006 : 
365 sqq.), est défendue l'idée que «l'étymologie » n'entre pas en ligne de compte dans le rapport entre le $\mathrm{Npr}$ et son référent ${ }^{15}$ - notons que ce que les auteurs nomment, de manière significative, «étymologie » (qui, donc, porte sur le passé), correspond, pour la plupart des Npr-coll, à leur sens compositionnel encore bien actuel. Transposons les termes présentés, avec un exemple : le Npr-coll Parti socialiste permet de référer au Parti socialiste, sans qu'on ait recours à son sens compositionnel.

Pour notre part, nous souscrivons, dans une certaine mesure, à cette conception : les Npr-coll peuvent référer sans le recours à l'interprétation de leur(s) composant(s) lexical(ux), et, s'ils sont polylexicaux, sans l'intermédiaire de leur sens compositionnel : la mémoire ou le contrat dénominatif mentionnés plus haut prennent le relais de l'interprétation une fois le Npr installé. Nous reprenons donc à notre compte ce que Bosredon (1997) nomme la «F-Nop» (Fonction de nomination propre) et qui, développée par l'auteur à propos des titres de tableaux, s'applique bien à notre objet. Au-delà de l'application stricte de la proposition initiale de Bosredon (qui ne considère pas les noms de groupes sociaux comme des Npr), nous interprétons ici la F-Nop comme décrivant le lien dénominatif stable entre un groupe humain institutionnel et son nom, qui s'instaure par création du nom et s'exerce par «contrat » et transmission. À cet égard, la F-Nop se rapproche de la notion de « désignation rigide » (Kripke, op. cit.).

Cependant, à la différence de Bosredon (op. cit. p. 234) qui découple, dans le processus de désignation, sens des expressions dénominatives et référence, il nous parait difficile d'être aussi général sur la question, complexe, de l'arbitraire, en disant que le caractère descriptif de l'expression ne joue pas de rôle. Dans un premier temps, repartons des exemples de Mill, Gardiner, Kripke, Coates : leurs arguments, appliqués aux Npr composés, ne font pas la différence entre les noms et les adjectifs (new (College), (Mont) blanc, Peak's Tunnel. Pourtant, le statut de Mont, de College, de Tunnel, et de Parti dans Parti socialiste sont spécifiques, puisqu'il s'agit de noms classificatoires (comme rue, jardin, cités par Jonasson et discutés par Bosredon) : dans les exemples cités, seul celui de Peak's Tunnel est probant pour l'argumentation défendue par les auteurs, puisque ce Npr réfère en contradiction avec la signification du nom tête classificatoire. À partir de là, tentons de résumer notre position : nous tombons d'accord avec Bosredon pour dire que « la notion de classification [cf. rue, jardin, mont, tunnel, parti] ne fournit pas à soi seule les conditions nécessaires et suffisantes pour construire la notion de dénomination propre [...] [et que] cette propriété est conférée par [...] F-Nop » (p. 236), i. e. l'instauration d'un lien dénominatif; mais en désaccord partiel quand il dit que «pour cela, [...] la mémoire du calcul identificatoire [doit] s'estomper » (p. 236). Selon nous, si effectivement la désignation peut être réussie sans passer par ce «calcul» (de fait, elle l'est, on l'a vu, pour Peak's Tunnel, et pour des Npr de partis comme Chasse, Pêche, Nature et tradition, Europe Écologie), cela ne signifie pas pour autant, pour les Npr-coll du moins, que la forme compositionnelle ne joue aucun rôle dans l'appariement Npr-référent, notamment pour la mémorisation, la transmission, le débat. En effet, si on ne se limite pas aux simples usages désignatifs des Npr-coll, et qu'on élargit la réflexion à leurs emplois métalinguistiques, argumentatifs, didactiques, ludiques, la forme choisie pour la dénomination (classificatoire ou non) n'est pas indifférente. Le caractère opératoire de la forme elle-même nous parait fort plausible sur le plan cognitif, et elle l'est aussi sur le plan des stratégies présidant au choix du nom et à sa mise en circulation, et sur le plan de son utilisation (cf. § 3).

\subsection{Pour conclure : noms de groupes sociaux, Npr ou pas Npr?}

En tout état de cause, il n'y a pas de raison de subordonner le statut de Npr à l'absence de motivation entre forme et référent : même si c'est peu perçu et peu utile pour la désignation, une bonne partie des Npr sont motivés, ou ont une étymologie transparente ${ }^{16}$. D'ailleurs, l'onomastique littéraire use de la motivation des noms de personnages ou de lieux de la fiction, sans qu'on renonce pour autant à les considérer comme des $\mathrm{Npr}^{17}$. En résumé, la motivation n'est pas nécessaire, mais pas non plus contradictoire avec le statut de Npr ; quand motivation il y a, celle-ci peut, ou non, être exploitée en tant que telle dans les emplois du Npr.

Sur la base des différents arguments avancés, et des caractéristiques des dénominations de groupes sociaux, nous proposons de les considérer comme des $\mathrm{Npr}$, lorsque le rapport signe-référent est fixé. 
Certes, il s'agit de Npr particuliers, dans la mesure où : ces noms désignent un groupe humain, ce qui a des incidences sur leur emploi en discours et sur leur forme ; ils sont forgés par ce groupe lui-même; ils sont construits avec du matériau lexical; le caractère motivé du nom, eu égard à l'existence du groupe, à sa raison d'être, à ses caractéristiques, parfois à son histoire, est plutôt la règle que l'exception.

Envisageons à présent le cas, évoqué plus haut, des dénominations conférées à un groupe instable ou en cours de constitution. Si l'on compare (avec des formes comparables) les Indignés, les precarios inflexiveis à les Verts du point de vue du contrat dénominatif, nous dirons que Les Verts est un Npr parce que le nom et son référent sont installés dans le paysage institutionnel, alors que les deux autres ne le sont pas (encore). Un des indices en est qu'ils ne prennent pas (ou pas toujours) de majuscule dans les textes. Dans les cas comme celui-ci, le contrat dénominatif nous paraît relever d'un processus diachronique : à la différence d'une ville, d'un pays, d'une personne ou encore d'un groupe social institutionnel et donc « officiel » dont le baptême (ou le changement de nom) est d'ordre binaire - le référent a $v s$ n'a pas tel nom -, c'est graduellement, de l'histoire et de leur reconnaissance sur la scène publique, que les groupes mentionnés ci-dessus recevront ce baptême, s'ils se stabilisent ${ }^{18}$. Ce processus complexe, où se mêlent auto- et hétéro-désignation (par les médias), dépasse le cadre linguistique, mais on peut supposer que la forme des dénominations y est pour quelque chose : il s'agit ici de syntagmes définis pluriel avec Nc désadjectival, dans lesquels les $\mathrm{Nc}$ ont une valeur prédicative («nous sommes/ils sont indignés/des indignés »), dont le processus menant au contrat dénominatif les abstrait. Quant au déterminant défini pluriel, il participe aussi de cette instabilité, entre ensemble d'individus et groupe constitué, cette fois (voir supra $\S 1.1$ ), puisque la forme plurielle oriente vers une appréhension distributive (omnis) alors même que ces éléments se constituent en entité-groupe.

Pour approfondir cette description, arrêtons-nous sur une autre dénomination de forme comparable : les Économistes atterrés est-il un $\mathrm{Npr}$, autrement dit le rapport dénomination-référent est-il «contractuellement» stabilisé ? On ne peut l'affirmer, puisqu'on constate une certaine instabilité formelle : le groupe se dénomme lui-même ${ }^{19}$ les Atterrés, le manifeste qui les a fait connaitre se nomme Manifeste d'Économistes atterrés, et le site porte la mention: "Nous sommes économistes et nous sommes atterrés ", qui explicite la dénomination du groupe. Selon nous, cette instabilité est à mettre au compte du référent (même s'il s'agit d'une association, les membres du groupe sont-ils considérés comme un groupe constitué ?), tout autant que de la forme [déterminant + nom classificatoire et adjectif qualificatif], qui affiche ce caractère intermédiaire.

Plusieurs paramètres seraient donc à suivre pour observer au fil du temps l'installation en Npr-coll d'une dénomination collective de la forme décrite :

- $\quad$ sur le plan linguistique, la majuscule graphique, sur tout ou partie de la dénomination ; maintien ou perte de la compositionnalité (par exemple le Npr-coll est-il reconnu et interprété en l'absence de l'un de ses constituants - les Atterrés ?) ; mais aussi dénomination de l'élément et modalités de l'appariement du nom de l'élément au Ncoll ;

- $\quad$ sur le plan (discursif et mémoriel) des emplois de la dénomination sur la scène publique, stabilisation de l'appariement Npr-référent.

\section{Forme des noms propres collectifs et enjeux discursifs}

Ce qui précède nous amène à aborder directement la question de la motivation des Npr-coll. À cet égard, nous considérons comme admis le fait que les Npr-coll, comme les autres Npr, peuvent (une fois stabilisés) référer sans recours au sens, et c'est maintenant sous un autre angle que nous les examinons : il s'agit de s'interroger sur le rapport entre la forme du Npr, le sens que construit cette forme, et le référent. Nous abordons cette question en trois temps : d'abord en proposant quelques éléments portant sur la stratégie du choix du nom ( $\S 3.1$ ), puis en relevant quelques types de motivations ( $\S 3.2$ ) ; nous en viendrons ensuite aux conséquences de la forme des Npr-coll sur le plan discursif $(\S 3.3)$. Alors que nous pouvions, jusqu'à présent, englober les quatre catégories de Npr-coll dans une même description, la problématique de cette section nécessite de détailler les spécificités de l'une ou de l'autre. 


\subsection{Des stratégies de nomination}

Nous ne ferons ici que de brèves remarques, destinées à introduire la suite en signalant quelques faits simples ou déjà établis, la question abordée ici relevant plutôt, sous certains aspects, de terrains de sciences politiques ou de sciences sociales.

Nous avons rapproché précédemment les Npr-coll d'autres noms, descriptifs ou potentiellement descriptifs : noms d'œuvres d'un côté, de rues ou de bâtiments de l'autre. Mais d'un point de vue pragmatique, ils s'en éloignent totalement; une comparaison avec les noms de marques et de produits parait plus adéquate : leur création fait l'objet de stratégies, et on peut dire que le rapport forme-contenu a une valeur performative. Comme le dit Bacot (2010) pour les noms de partis :

Le nom propre n'est politique que s'il n'est pas arbitraire, que s'il est porteur d'un message, que s'il ne se contente pas de désigner l'objet qu'il présente comme unique, mais dit quelque chose à son sujet - quelque chose qui lui fait jouer un rôle dans la conflictualité sociale.

Il faut donc envisager la signalétique des Npr-coll dans le cadre de stratégies de création ou d'emploi du nom. Les buts qui sous-tendent ces stratégies sont sensiblement différents selon les types de groupes : les partis politiques, par exemple, s'adressent à l'ensemble d'une population, à la différence des syndicats, dont le rassemblement s'exerce sur le terrain professionnel. Quant aux associations et aux groupes culturels, a fortiori, ils n'ont pas d'objectif de cet ordre en termes de "rassemblement » et de visibilité. Pour tous les groupes sociaux cependant, le choix du nom et du message qu'il porte répond à un besoin d'affichage en externe (vis-à-vis du «monde», médiatique, politique...) et en interne (vis-à-vis des membres du groupe), affichage qu'on pourrait dire dans les deux cas «identitaire - fût-il raté, ou opaque pour le commun des mortels.

Attardons-nous un moment sur le cas des partis : dans leur cas, puisqu'ils se situent sur le terrain de la nation, l'affichage identitaire doit apporter aussi une différenciation - une ressemblance, une continuité -, avec les autres partis ${ }^{20}$. C'est ce que montre la description du nom Union des Démocrates et Indépendants par Bacot et Barbet (2013), qui commentent chacun des lexèmes le composant par rapport à ceux d'autres noms de partis. On pourrait ajouter à cette analyse la question : «Indépendants, oui, mais de quoi ? », qui se pose aussi ailleurs, car on trouve cet adjectif dans d'autres Npr de partis (Parti Ouvrier Indépendant), comme s'il devait introduire une valeur ajoutée. Les adjectifs nouveau (Le Nouveau Centre, Nouveau Parti Anticapitaliste), radical (Parti Radical de Gauche) peuvent aussi être interprétés comme répondant à cette nécessité d'afficher continuité et/ou différenciation. Il y a là, sur le plan linguistique et sur celui de l'histoire des partis, des faits d'ordre relationnel qui complexifient la motivation éventuelle de ces Npr.

D'une manière générale donc, même dans les cas où elle n'est pas aboutie ou pas réussie, dans les cas où elle est obscure ou périmée, la motivation de la dénomination est un enjeu : enjeu descriptif (nécessité d'intelligibilité, pour les médias et le public), enjeu identitaire, référence à d'autres groupes, appel à la connivence (voir l'antiphrase dans Sauvons les Riches).

\subsection{Forme des noms propres collectifs et motivation}

Nous abordons à présent la motivation sous un angle linguistique, en ne nous attachant qu'à ce que la forme des Npr-coll est susceptible de donner à voir ou à imaginer, sans entreprendre d'enquête sur le référent. Dans ce cadre, "motivation» est la notion générique, qui se spécifie en termes de « descriptivité » (éventuelle), laquelle est compositionnelle (dans les cas de polylexicalité (i)) ou non (un seul lexème (ii)). Enfin, dans certains cas, la motivation doit plutôt s'envisager en termes d'« évocation » (iii). Cet aspect ne se situe pas sur le même plan que les autres, mais est plutôt transversal.

Dire que le Npr permet de référer directement ne signifie pas que l'expression dénominative elle-même n'a pas de sens ; les Npr-coll sont à cet égard un exemple emblématique. Du fait qu'ils sont forgés à partir de matériau lexical dans le but de, sinon coller à la réalité qu'ils dénomment, du moins y renvoyer de manière lisible, ils ne peuvent en effet pas être dits « vides de sens ». C'est ainsi que s'établit, selon nous, 
un rapprochement entre deux perspectives distinctes présentées en 2.2. : le Npr comme description de son référent d'un côté, et le caractère descriptif de l'expression de l'autre. Ce rapprochement n'est cependant nullement direct, dans la mesure où les cas où l'on peut parler de "descriptivité » pure et simple (Parti socialiste $=$ parti + socialiste $)$ sont rares, la motivation prenant différents chemins, plus complexes. À partir d'éléments du relevé de 1.2., nous en proposons une interprétation selon quelques grandes lignes il ne s'agit ni d'entreprendre une typologie, ni même d'épuiser le potentiel de nos exemples.

Forgés à partir de lexèmes ou à partir d'autres Npr (Indochine, Institut Montaigne, Europe Écologie, compagnie Pietragalla), les Npr-coll reposent, par la F-Nop, sur l'établissement d'un rapport inédit entre ce matériau, lui-même arbitraire ${ }^{21}$, et un nouvel objet. C'est donc fondamentalement dans l'utilisation de ce matériau qu'intervient la question de la motivation. Celle-ci se différencie ensuite en termes de Nprcoll polylexical vs monolexical. Dans le premier cas, qui est le plus fréquent de notre relevé et qui représente la totalité des $\mathrm{Npr}$ de partis, la question de la motivation se pose d'abord en termes de transparence compositionnelle. En effet, tout en étant figées, les dénominations ne sont pas opaques ${ }^{22}$. Dès lors, ce sont les types de lisibilité - description, et de quoi ? évocation ? - qu'il faut préciser.

(i) Polylexicaux. Les Npr-coll qu'on qualifiera, dans un premier temps, de strictement « descriptifs » (essentiellement parmi les catégories 1,2 et 3 du $\S$ 1.2.) sont de la forme [Ncoll classificatoire (+ épithète) + expansion(s) (épithète, complément de nom)] - Centre National des Indépendants et Paysans, Ligue Communiste Révolutionnaire, Parti de Gauche, Confédération Paysanne, Mouvement des entreprises de France, Cercle des Économistes, Ligue des Droits de l'homme etc. -, l'expansion venant généralement préciser la catégorie ou l'identité, en particulier en termes d'appartenance ou de ligne politique $^{23}$. Nous ne nous y attardons pas (cf. Tournier 1981). On peut aussi dire descriptifs les Npr-coll de la forme [nom au pluriel + expansion] - Médecins du Monde/sans frontières, (les) Petits chanteurs à la Croix de Bois -, descriptifs dans la mesure où le pluriel ainsi que le but du rassemblement ou l'identité sont marqués lexicalement. Cependant, même dans des cas apparemment simples de lisibilité componentielle, la perspective en termes de «description » du référent est à regarder de près : comment par exemple interpréter les expansions du Monde (Médecins du Monde), populaire (UMP), pour la France (Mouvement pour la France), national (Front National), républicain et citoyen (Mouvement Républicain et citoyen) ? On ne peut pas réellement parler de «description » : il s'agit en réalité d'un autre type de motivation, plus complexe. On aborde ici le domaine de l'évocation, par essence sans limites, souvent incernable, et susceptible de concerner tous les Npr-coll : évocation renvoyant à des valeurs, des qualités, des symboles, dans les exemples de ce paragraphe (voir aussi croix de bois)... Nous y revenons plus bas (iii).

Certains autres Npr-coll peuvent être dits descriptifs, mais de manière indirecte : avec les expressions nominales Droit au logement, Action contre la faim, Amnesty International, Secours populaire/catholique, Lutte ouvrière, Force ouvrière, Chasse, Pêche, Nature et Tradition, les phrases Que choisir? Sauvons l'Université, ce sont, par des biais syntaxiques différents, à la fois des programmes et des appartenances qui sont décrits. Dans Occupy Wall Street, le Npr décrit une action et un lieu, mais aussi renvoie à (décrit ?) la finalité de cette action. On remarque dans tous ces cas que le caractère collectif du référent n'apparait pas, ou de manière très indirecte, en termes de but commun.

(ii) Monolexicaux. La description du groupe et/ou de son objectif ne peut pas être atteinte de la même façon avec les noms monolexicaux. On trouve des cas qui en relèvent parmi les $\mathrm{Npr}$ de syndicats (Autonomes, Alliance), de partis (les Verts), d'associations et organisations (les Anonymous, les Indignés), et de groupes culturels (les Innocents, the Doors). Leur lisibilité éventuelle peut être abordée sous différents angles : a) la plupart d'entre eux reposent sur une conversion désadjectivale (Autonomes, Verts, Anonymous) - on peut leur appliquer l'analyse " prédicative » proposée supra à propos de les Indignés; b) le caractère collectif du référent apparait dans certains avec la marque du pluriel et le déterminant; c) leur sens lexical renvoie à un programme (Alliance, Autonomes), à une «identité » (les Indignés, les Anonymous), à une symbolique (les Verts, peut-être est-ce le cas de Fauve?) ou à... des questions, comme avec beaucoup de noms de groupes musicaux, qui jouent plus sur une connivence (éventuelle) et ne paraissent pas fondés sur une recherche de lisibilité descriptive. Ainsi Fauve (de nouveau) renvoie-t-il 
peut-être, métonymiquement et métaphoriquement, au fauve (l'animal) et à son caractère, ou peut-il faire référence à l'école de peinture (le fauvisme)...

(iii) En effet, absence de lisibilité ne signifie pas absence de motivation. Nous avons parlé plus haut d'《évocation», faute d'un terme vraiment adapté qui englobe cet autre aspect de la motivation, complémentaire de la «descriptivité » et qui s'y ajoute souvent - cf. les cas décrits sous (i) : dans nos exemples, il peut s'agir de connotation, mais aussi de références partagées; dans tous les cas, et à la différence de la « descriptivité », l'interprétation (si elle est possible) repose sur des inférences.

Examinons ceci. Étant donné la diversité des cas et, surtout, le caractère indécidable de la plupart, nous nous contenterons de proposer quelques exemples et quelques conjectures. a) Il nous semble que l'évocation de références n'est pas du même ordre pour toutes les catégories de Npr-coll. Besoin de sérieux dans les trois premières, ouverture à la fantaisie dans la quatrième; b) les chemins de l'évocation sont certes divers, mais certains peuvent être cernés : référence à une personne (Institut Montaigne, Fondation Copernic), à un événement (Jeudi Noir renvoie à la crise de 1929, Emmaüs à un événement biblique), à des valeurs positives, mais de différents ordres (Synergie pour un syndicat, Intermède pour un groupe de loisirs), à des symboles (Croissant-Rouge, Croix-Rouge); c) l'évocation peut à son tour être le déclencheur d'une motivation - on peut voir les membres des syndicats Synergie et Alliance selon les qualités que ces noms évoquent; on peut jouer à se représenter les Beatles comme des «scarabées »; d) la matérialité linguistique, le jeu sur les sonorités (peu présent dans nos exemples, mais susceptible de se développer ${ }^{24}$ ) est une autre voie d'évocation: on la trouve exploitée dans la paronomase Robin des Toits (où toits a aussi une part de descriptivité) ou dans le mot-valise Beatles, motivé avec beat. Enfin, on ne peut exclure la création purement ludique, dépourvue de motivation : que comprendre par exemple de Chanson plus Bifluorée? de Tryo (en dehors de l'homophone trio)? Mais ces Npr aussi peuvent déclencher une recherche de motivation, tant est forte la «pulsion de sens » (comme le dit Genette $o p$. cit.), et singulièrement avec les Npr-coll.

\subsection{Forme des noms propres collectifs et emploi en discours}

Coates (op. cit.) propose de distinguer les deux perspectives que sont l'«octroi » (bestowal) des Npr et leur usage. Du point de vue de l'octroi (l'acte de baptême), il les considère comme vides de sens (senseless), mais non pas du point de vue de leur usage (ils ne sont pas meaningless). Nous avons présenté dans ce qui précède notre option concernant «l'acte de baptême » des Npr-coll, distincte de celle de Coates. Nous nous intéressons à présent à leur emploi en discours, en considérant que ces deux aspects sont liés, étant donné le type de référent dénommé par les Npr-coll (référent collectif et institutionnel) et donc le caractère public du nom et du référent, étant donné aussi le fait que le nom est créé en donnant, ou pour donner, une information directe ou indirecte sur le référent (cf. § 3.2).

Notre proposition s'ancre dans une conception pragmatique du Npr qui tient compte des enjeux dans lesquels il entre (cf. § 3.1.), et selon laquelle l'emploi strictement désignatif n'est qu'un emploi parmi d'autres (fût-il le plus fréquent, ce qui reste à démontrer pour les Npr-coll) : le Npr-coll est toujours susceptible d'être objet de discours (en mention), ou objet de jeu; sa forme, les éléments qui le composent, son sens, compositionnel ou non, peuvent être repris en tant que tels dans des débats, des présentations, des explications... Témoins, les sites internet de la plupart des groupes sociaux mentionnés (catégories 1 à 3 et parfois 4), qui explicitent tout ou partie de leur nom, ce qui est d'ailleurs sans doute une nécessité pour les Npr de partis : le Parti Ouvrier Indépendant justifie ouvrier, le Centre national des Indépendants et Paysans discute indépendant. Témoins aussi, certaines discussions lors de créations de "mouvements» sur le choix du Ncoll classificatoire: "nous nommerons-nous mouvement, réseau, union, collectif...? ». Témoin enfin, l'emploi en mention d'éléments d'un $\mathrm{Npr}$ de parti dans certains genres journalistiques (chroniques ou éditoriaux) : actuellement, on rencontre souvent l'adjectif socialiste entre guillemets et employé en connotation autonymique, c'est-à-dire en usage et en mention (ReyDebove 1997) - le parti "socialiste ». Cet emploi modalisant sert, de manière économique, les argumentations traitant de contradiction et de reniement. 
Nous venons de mentionner brièvement des cas où la forme des Npr-coll est susceptible de jeu ou de discussion, de par la signification lexicale de certaines parties de l'expression ou de par la composition du nom. Nous terminons à présent par un autre aspect, tenant plutôt à la sémantique du collectif en tant que tel : celui du double niveau mentionné en $\S 1.1$, niveau du tout (groupe constitué, totus) et des éléments dans leur pluralité (omnis). Tous les Npr-coll sont par définition concernés, mais la forme de certains induit davantage d'effets associés, en termes d'ambigüité, d'hésitation ou de jeu : il s'agit, en particulier, de Npr-coll dont la forme est au pluriel (les Verts, les Indignés, les Têtes Raides, les Innocents etc.) et de ceux pour lesquels la présence du déterminant pluriel s'avère être fluctuante ((les) Anonymous), (les) Occupy). Dans ces cas comme dans les autres, l'expression est susceptible de désigner le groupe constitué et les éléments, mais, à la différence de Npr-coll débutant par un Ncoll classificatoire (parti, cercle, ligue, union etc.), cette expression oriente de manière privilégiée vers les éléments. Il y aurait ici beaucoup de sillons à creuser (sens lexical, mode de nomination d'un membre, e. g. un vert, stabilité de l'instauration de la F-Nop etc.). Nous nous en tenons à quelques exemples.

Exemples de fluctuation selon qu'on désigne le tout ou les membres :

(5) Anonymous Belgique veut un internet libre. (site de Anonymous Belgique)

(6) Le 23 Février 2013 lors d'une manifestation des Anonymous dans une rue de Nottingham en Angleterre [...].

Exemple de clin d'œil (ou de maladresse) ; voir aussi (2) :

(7) Le $100^{\text {ème }}$ anniversaire des Petits chanteurs à la croix de bois [...] Les Petits chanteurs à la croix de bois ont 100 ans. Émission hommage [...] (Télérama, 20/12/06).

Les jeux de mots avec les Verts sont fréquents, et on invoque alors le sémantisme de l'adjectif de couleur. Mais le caractère collectif du nom et la forme plurielle interviennent aussi, comme en (8) où sens lexical et pluralité se combinent :

(8) Les Verts ne sont toujours pas mûrs. (France Inter, revue de presse 2006)

Variations formelles, hésitations et ambigüités, jeux de syntaxe et de sens révèlent les caractéristiques de ces Npr-coll. Selon nous, leurs propriétés sémantiques et référentielles, certes atypiques au regard des autres Npr, mais régulières au sein de cette catégorie, ne s'opposent pas au statut proprial des dénominations, pas plus que l'éventuelle motivation de leur forme.

Plus généralement, la forme des Npr-coll, surtout lorsqu'ils sont polylexicaux ou composites, induit en elle-même des variations (flexion ou omission du déterminant lorsqu'il y en a un, désignations elliptiques ou siglées...), lesquelles variations ne nuisent pas pour autant au maintien du contrat dénominatif.

\section{Conclusion}

Nous avons proposé ici un défrichage, d'un point de vue sémantique, discursif et formel, d'une catégorie de dénominations, les dénominations de groupes sociaux constitués. Leurs caractéristiques sémanticoréférentielles (le référent est composé d'une pluralité d'éléments humains, rassemblés en un groupe selon un but commun), leur forme, et la signification qui s'attache à cette forme (souvent compositionnelle et, dans tous les cas, basée sur du matériau lexical), mais aussi la vocation publique de ces dénominations et de leur référent en font des objets linguistiques singuliers. Ces caractéristiques ne s'opposent pas, selon nous, à ce qu'on classe ces dénominations parmi les noms propres, en tant que noms propres collectifs, et elles présentent aussi suffisamment de points communs pour qu'on regroupe ces Npr-coll sous une même description.

Plusieurs de leurs singularités nous paraissent riches d'implications : la formule « acte de baptême », ou encore «octroi du nom», prend ici un sens particulier, celui d'une nomination pesée et choisie collectivement, en fonction de ses implications sémantiques et discursives - présence du référent et du nom sur la scène publique et donc stratégies en termes de lisibilité, de mémorisation (dont nous avons peu 
parlé), de transmissibilité, voire de « désirabilité ». Nous avons également envisagé (cf. les Indignés, les Économistes Atterrés) le cas où cet acte de baptême s'opère en partie de l'extérieur (par les médias), et progressivement. Nous en tirons l'idée que la notion d'acte de baptême reçoit ici un sens particulièrement fort, d'un point de vue social (nommer un groupe par un Npr-coll, c'est assurer son statut de groupe stable), et l'observation que, dans cet acte, s'exercent des influences croisées : celles de la langue (présence du déterminant, signification prédicative et/ou classificatoire du-des lexème-s), celles impliquées par les caractéristiques du référent, celles enfin des conditions (au sens large) d'emploi du nom.

Nous avons essentiellement mis en exergue des ressemblances entre les catégories de Npr-coll étudiés, soit du point de vue de leurs caractéristiques, soit du point de vue des questions qu'ils soulèvent (statut proprial, descriptivité ou, plus généralement, motivation), mais de nombreuses pistes complémentaires restent à explorer : analyse, du point de vue morphosyntaxique, de l'emploi de différents types de Nprcoll (variation du déterminant, phénomènes d'accord) : les analyses faites sur les $\mathrm{Ncoll}$ ( $\mathrm{N}$ communs) sont connues (voir Lammert 2010), mais la polylexicalité des Npr-coll ne peut qu'induire d'autres phénomènes; description particulière de chacune des quatre catégories de Npr-coll voire d'autres, du point de vue de sa « signalétique », en la mettant en rapport avec ses particularités sur la scène publique ; rapport entre la forme du Npr-coll (par exemple monolexical ou polylexical) et sa mise en circulation dans les discours, sa transmission, sa mémorisation; rapport entre la forme du Npr-coll et son potentiel argumentatif, en lui-même (Ligue pour [...]; Parti de Gauche (vs autres partis « de gauche »)), mais aussi selon les argumentations où on le fait entrer; nom donné à l'élément (un Vert, un socialiste, ? un Emmaüs, ? un Médecin du Monde), variation de ce nom et stabilité du rapport dénominatif; enfin, rapport, esquissé ici, entre des traits systématiques des Npr-coll (forme et sens), ou de tel d'entre eux, et les jeux de mots qu'on peut en faire.

Nous avons observé à chaque pas que la description des Npr-coll nécessite les perspectives croisées de plusieurs champs de la linguistique et de l'analyse des discours, dans la mesure où ces noms, de par leur caractère institutionnel et public, sont créés et circulent, certes avec le matériau d'une langue donnée, mais aussi dans le cadre d'une société donnée, et que ces déterminations interagissent. En ceci, notre étude linguistique s'ouvre sur d'autres études, en analyse du discours et en sciences sociales.

\section{Références bibliographiques}

Bacot, B. et Barbet, D. (2013). Indépendants. Le retour. Notes de recherche d'actualité. Mots - Les langages du politique, en ligne [http://icar.univ-lyon2.fr/revue mots/], page consultée le 5/09/2013.

Frath, P. (2010). La référence par le nom: vers une linguistique anthropologique. In Frath, P., Pauchard, J. \& Lansari, L. (coord.) Res per Nomen 2 : langue, référence et anthropologie. Reims : EPURE.

Bacot, P. (2010). Développement et diversification d'une onomastique politique. Mots. Les langages du politique, 94, 47-56.

Barthes, R. ([1967] 1972). Proust et les noms. In Le degré zéro de l'écriture. Paris : Éditions du Seuil.

Bosredon, B. (1997). Les titres de tableaux : une pragmatique de l'identification. Paris : PUF.

Bosredon, B. (2011). Dénominations monoréférentielles, figement et signalétique. In Anscombre, J.-C. et Mejri, S. (éds) Le figement linguistique : la parole entravée. Paris : Honoré Champion, 155-170.

Calabrese, L. (2010). Le rôle des désignants d'événements historico-médiatiques dans la construction de l'histoire immédiate. Une analyse du discours de la presse écrite. Doctorat, Université Libre de Bruxelles.

Cislaru, G. et Lecolle, M. (2010). Noms propres de lieux habités, espace et temporalité. Revue de Sémantique et Pragmatique 25-26, 121-137.

Coates, R.A. (2006). Properhood. Language Vol.82, $\mathrm{n}^{\mathrm{o}} 2$, 356-382.

Ferret, S. (2011). L'identité. Paris : Flammarion. 
Gardiner, A. ([1953] 2005). [The Theory of Proper Names] Théorie des Noms Propres. Essai polémique. Modèles Linguistiques tome XXVI-1, vol. 51, 55-112.

Genette, G. (1976). Mimologiques. Voyage en Cratylie. Paris : Éditions du Seuil.

Gouvard, J.-M. (2000). Poétique des noms propres. In Gouvard, J.-M. et Murphy, S. (éds) Verlaine à la loupe. Paris : Champion, 159-184.

Gross G. et Massoussi T. (2011). Figement et transparence. In Anscombre, J.-C. et Mejri, S. (éds) Le figement linguistique : la parole entravée. Paris : Honoré Champion, 95-108.

Jonasson, K. (1994). Le nom propre. Constructions et interprétations. Louvain-la-Neuve : Duculot.

Kleiber, G. (1981). Problèmes de référence : descriptions définies et noms propres. Metz : Recherches linguistiques.

Kripke, S. ([1972] 1982). [Naming and Necessity] La Logique des noms propres. Paris : Minuit.

Kristol, A. M. (2002). Motivation et remotivation des noms de lieux : réflexions sur la nature linguistique du nom propre. Rives 11, Récit et toponymie. http://rives.revues.org/document121.html, page consultée le 7 mars 2014.

Lammert, M. (2010). Sémantique et cognition : les noms collectifs. Genève-Paris : Droz.

Lecolle, M. (2011). Caractérisation des entités nommées du point de vue du rapport élément/collection. Le cas des 'noms propres collectifs'. Journée ATALA, Paris, 20 juin 2011. [Présentation en ligne] http://tln.li.univtours.fr/Tln_Colloques/Tln_REN2011/Lecolle-ATALA-20juin2011.pdf, page consultée le 6 mars 2014.

Lecolle, M. (2013). Noms collectifs humains : un point de vue de sémantique lexicale sur l'identité dans le rapport individu/groupe. Revue ¿Interrogations? 16, [en ligne] http://www.revue-interrogations.org/NOMSCOLLECTIFS-HUMAINS-UN-POINT, page consultée le 7 mars 2014.

Lecolle, M. (à paraître a), 2014). Noms propres institutionnels: référence individuelle et référence collective. $A C T A S$ XXIV CONGRESO ICOS BARCELONA.

Lecolle, M. (à paraitre b), 2014). «Nom propre de lieu habité : polyvalence et polysignifiance ». Actes du colloque rennais Défis de la toponymie synchronique.

Leroy, S. (2004). Le nom propre en français. Gap-Paris : Ophrys.

Leroy, S. (2011). Catégories et identités. Mémoire de synthèse d'habilitation à diriger des recherches, Université Paris Ouest Nanterre La Défense.

Rey-Debove J. ([1978] 1997). Le métalangage. Étude linguistique du discours sur le langage. Paris : Armand Colin.

Mill, J. S. ([1843] 2005). Noms connotatifs et non-connotatifs, Modèles Linguistiques tome XXVI-1, vol. 51, pp. 3545. [Traduction de Louis Peisse], section 5 du chapitre II livre I, System of Logic.

Searle, J.R. (1972). Les actes de langage. Paris : Hermann.

Tournier, M. (1981). Vers une grammaire des dénominations socio-politiques au début de la Troisième République (1879-1905). Mots 2, 51-72.

Vaxelaire, J.-L. (2005). Les noms propres. Une analyse lexicologique et historique. Paris : Honoré Champion.

Veniard, M. (2009). La dénomination propre la guerre d'Afghanistan en discours : une interaction entre sens et référence. Les Carnets du Cediscor 11, 61-76.

Wilmet, M. (1988). Arbitraire du signe et nom propre. Annexes des Cahiers de linguistique hispanique médiévale, volume 7, Hommage à Bernard Pottier, 833-842.

\footnotetext{
* Tous mes remerciements à Brigitte Wiederspiel, ainsi qu'à mes relecteurs anonymes pour leurs précieuses suggestions, qui m'ont permis d'améliorer ce travail.

${ }^{1}$ Lecolle (2013). Notre présentation reste rapide et se limite à l'essentiel de ce qui sert le propos de l'article. Pour des compléments sur les Ncoll, voir principalement Lammert (2010).
} 


\begin{abstract}
${ }^{2}$ Extrait rapporté par Ferret (2011: 115). Pour une approche syntaxique et morphosyntaxique des Ncoll, voir notamment Lammert (2010) et les auteurs qu'elle cite.

${ }^{3}$ Nous prenons directement et par anticipation des exemples de Npr-coll, mais l'observation est similaire avec des Nc collectifs. Quant à les Verts en (2), il s'agit pour nous, malgré sa forme plurielle, d'un Npr-coll ; nous avons discuté dans Lecolle (à paraitre a) cette apparente contradiction.
\end{abstract}

${ }^{4}$ Alors que, pour les couples LCR/NPA et CNPF/MEDEF, le second remplace le premier, les associations Médecins sans Frontières et Médecins du Monde coexistent : celle-ci fut créée en 1980 d'une scission au sein de Médecins sans frontières.

${ }^{5}$ Nos sources sont principalement la presse d'information générale et la presse culturelle (en ligne, papier, radio). Notre collecte provient aussi pour l'essentiel des sites suivants : "France politique » tenu par le journaliste politique de La Croix Laurent de Boissieu (http://www.france-politique.fr/) pour les noms de partis, "nonfiction.fr Le quotidien des livres et des idées » (http://www.nonfiction.fr/home.htm) pour d'autres noms de groupes (think tank) et Wikipedia (https://fr.wikipedia.org/wiki/Accueil).

${ }^{6}$ Nous nous basons sur une connaissance «moyenne » des affaires publiques, telle que celle d'un citoyen informé, mais non spécialiste, ce qui veut dire que, d'une part, nous ne présentons que quelques noms (les plus connus en France), d'autre part, nous n'entreprenons pas d'enquête sur les référents des noms, mais nous intéressons à ce qui en est affiché publiquement : sites internet, presse, participation à des manifestations publiques (tracts, déclarations).

${ }^{7}$ Cf. Lecolle 2011. Dans Lecolle (à paraitre a), nous comparons entre eux plusieurs « Npr collectifs », dont les noms d'entreprise; nous distinguons ceux-ci des Npr-coll étudiés ici sur la base du lien entre le tout (l'entreprise) et l'élément (dont on ne peut pas dire qu'il en est un «membre»- mais plutôt un employé, salarié etc.). Une autre différence apparait, liée à la première : dans le cas de l'entreprise, son Npr n'est créé ni par ni pour les «membres ».

${ }^{8}$ Voir dans Lecolle (2011) le tableau récapitulatif p. 18, qui reprend les cas discutés ici, ainsi que celui du nom de continent (l'Europe).

${ }^{9}$ Présentés en ordre alphabétique, par commodité. Avec ou sans déterminant, selon qu'il appartienne ou pas au nom (nous confirmons avec les sites des partis eux-mêmes). Avec ou sans sigle, selon qu'il est, ou non, usuel. Les italiques signalent le nom, et éventuellement le sigle. Nos éventuelles incises sont en style «normal ».

${ }^{10}$ Le MEDEF a remplacé à la fin des années 90 le CNPF (Conseil National du Patronat Français). Ce nom portait explicitement la marque de l'appartenance des membres (patronat). L'information est plus indirecte avec $M E D E F$ (entreprises).

${ }^{11}$ Comme leurs noms ne l'indiquent pas, il s'agit de groupes français de jazz.

${ }^{12}$ Voir Leroy (2004), Jonasson (1994), Vaxelaire (2005). Gardiner (2005 : 75-76) aborde la question du statut de ces noms, mais on ne voit pas clairement quelle est sa réponse. Bosredon les considère comme des "dénominations propres ", mais pas comme des Npr. À la suite de Tournier (1981), seul Bacot (2010) s'attaque directement au développement d'une « onomastique politique », centrée sur les noms de partis.

${ }^{13}$ Dans Lecolle (à paraître), nous développons la distinction entre l'appréhension en discours des éléments (omnis) et du groupe constitué (totus), et décrivons des éléments de contexte qui orientent vers l'une ou l'autre des interprétations.

${ }^{14}$ Voir des synthèses chez Vaxelaire (2005), Calabrese (2010), Leroy (2011) notamment. Pour une présentation approfondie des approches logiques et sémantiques du Npr, voir Kleiber (1981).

${ }^{15}$ Le New Collège n'est plus «new » et Peak's Tunnel ne désigne pas un tunnel. Quant aux autres : que Dartmouth soit à l'embouchure de la Dart, que le Mont Blanc soit blanc (enneigé) ou non, peu importe pour le lien dénominatif nom-référent.

${ }^{16}$ On trouve dans le TLFi (http://atilf.atilf.fr/tlf.htm) 150 noms de villes se terminant par ville, et 90 par bourg.

${ }^{17}$ Voir Barthes (1972), Genette (1976), Wilmet (1988), Gouvard (2000), ainsi que Kristol (2002) pour les toponymes.

${ }^{18}$ L'exemple contemporain (2013) de la dénomination «Les Amis de la Syrie », employé dans la presse avec ou sans modalisation, serait à observer sous cet angle : deviendra-t-il une dénomination propre, voire un nom propre ?

${ }^{19}$ Cf. son site : http://www.atterres.org/ 


\footnotetext{
${ }^{20}$ Continuité/ressemblance qu'on trouve aussi avec d'autres organismes, e. g. Croissant rouge/Croix Rouge, cf. $\S 1.2$.

${ }^{21}$ Nous n'envisageons pas les cas de motivation phonique ou graphique. Pour un tour d'horizon érudit des différents aspects de la motivation (du « mimologisme »), voir Genette (1976).

${ }^{22}$ L'opacité n'est en rien un critère définitoire du figement, même si elle l'accompagne souvent (Gross et Massoussi 2011 : 101). Voir aussi Bosredon (2011) pour les dénominations polylexicales.

${ }^{23}$ Le vocabulaire politique lui-même est très certainement soupesé (cf. § 3.1), et historiquement marqué.

${ }^{24}$ Un exemple : le jeu des sigles mentionné en $\S 1.2$. pour CAP21 est basé sur la matérialité de l'expression. Des entreprises de « créatifs » proposent leurs services pour forger des Npr, ce qui pourrait aussi concerner les partis.
} 